\title{
The recency effect in pigeons' long-term memory
}

\author{
DAVID R. THOMAS, THOMAS B. MOYE, and ERIC KIMOSE \\ University of Colorado, Boulder, Colorado
}

\begin{abstract}
Four experiments are reported in which pigeons first learned one wavelength discrimination (green $\mathbf{S}+$, yellow $\mathbf{S}-$ ) and then the reversal; finally, after various delays, they were tested for wavelength generalization in extinction. In Experiment 1, the two problems were learned in different contexts; testing in Context 1 produced maximal responding to green in only half of the subjects, even when testing was delayed 30 days. In Experiment 2, testing of the subjects repeatedly in both contexts showed good control by each context after a 30-day delay. In Experiment 3, both problems were learned in the same context, and all gradients showed recency, peaking at yellow, even after 30 days. In Experiment 4, the subjects learned a series of reversals in the same context, terminating in yellow, S+, green, S-, and their gradients peaked at yellow, even after a 30-day delay. In Experiments 3 and 4, the gradients became flatter with increasing delays, and they were flatter in Experiment 4 (after three reversals) than in Experiment 3 (after one reversal). The location of the peak was not affected by delay, but only by testing in a context that had been uniquely associated with Problem 1 (Experiments 1 and 2). It is proposed that the location of gradient peaks indicates what is being remembered, whereas the slope of the obtained gradients indicates how well the target memory has been retrieved.
\end{abstract}

In the study of proactive interference (PI) in human memory, experimental subjects learn two conflicting or incompatible habits in sequence. They are later instructed, at the time of a memory test, to respond in accordance with the terminal habit, and their intent to comply with these instructions is taken for granted. To the extent that they perform more poorly than do subjects trained only on the terminal habit, a process of PI is inferred.

In investigating PI in nonverbal subjects (e.g., rats or pigeons), an alternative to verbal instructions is required such that the subjects' intent to perform according to most recent contingencies can reasonably be inferred. Fortunately, it has been well established that when trained in sequence on two conflicting habits, animals will generally respond appropriately to the last learned habit, particularly if the test is carried out soon after the completion of training (cf. Gleitman, 1971; Spear, 1978; Thomas, 1981). This recency effect is so ubiquitous that it may be interpreted as a reflection of the subjects' "intent" to "do what they did last." To the extent that subjects trained on a previous task perform the lastlearned habit less well than do subjects trained only on the terminal habit, particularly if a delay interval has intervened, PI is inferred.

We thank Paul Beck for assistance in the running of subjects. This paper was presented at the April 1983 meeting of the Rocky Mountain Psychological Association in Snowbird, Utah. Requests for reprints should be addressed to David R. Thomas, Department of Psychology, Campus Box 345, University of Colorado, Boulder, CO 80309.
Spear (1971) reported a series of studies in which rats were first trained on a passive avoidance task, then taught active avoidance, and subsequently tested, without reinforcement, either immediately or after a delay period. In the immediate test, the rats showed a strong recency effect, responding appropriately to the active avoidance contingency, and indistinguishably from subjects who had learned only the active avoidance response. In the delayed test, however, the rats showed interference, often responding with the long latencies characteristic of passive avoidance behavior. This change in behavior over time did not happen to subjects trained only on an active avoidance task. Spear also showed that the amount of PI obtained could be increased by training the initial passive avoidance response in the presence of a distinctive context, for example, a tone, and then testing after a delay in the presence of that tone.

Thomas (1981) described a series of studies of longterm memory in pigeons, in which measures of retention and forgetting were derived from stimulus generalization gradients. He argued that forgetting might be reflected in a loss of stimulus control, operationally defined as a flattening of the generalization gradient, even when measures of response rate showed no effect. If the two conflicting tasks used in a study of PI involve responding to different stimuli, the strength of the two habits can be (relatively) independently assessed if the generalization test series includes both training stimuli.

In an experiment conceptually similar to the Spear's (1971), which used the presence of a tone as a distinc- 
tive context, Thomas, McKelvie, Ranney, and Moye (1981) first trained pigeons to perform a successive wavelength discrimination (e.g., peck green and not yellow) and then trained them on the reversal (peck yellow and not green). On the day after the completion of training on the reversal task, a wavelength generalization test was carried out in extinction. Despite the 24-h delay, the gradients still revealed recency, with maximal responding occurring to the yellow stimulus. On the other hand, there was evidence of PI in that the gradients were flatter than those of a control group which had learned only the second (green $\mathrm{S}-$, yellow $\mathrm{S}+$ ) problem.

One group in the Thomas et al. study learned the original problem in one context, houselight and tone (HL T), and the reversal problem in a different context, houselight-off and white noise ( $\overline{\mathrm{HL}} \mathrm{N})$. When testing was carried out in Context 1 (HL T), PI was substantially increased and recency was entirely overcome. No subjects' gradients peaked primarily at yellow. Some peaked at green, that is, appropriately to the test context, while others peaked at both green and yellow. Like Spear's (1971) rats, the pigeons in this group seemed to be confused by conflicting "instructions" - the implicit recency instruction, "Do what you did last," and the more explicit contextual instruction "Do what you did in this context." The contextual instruction provided by Context 1 canceled the recency instruction but was not compelling enough to result consistently in behavior appropriate to the initially learned task. One possible reason for this was that the context was not adequately associated with the original problem. This interpretation was ruled out in a second experiment in which training was done as in Experiment 1 but wavelength generalization testing was carried out with the two contexts alternating during the test session. For every subject, the gradient obtained in each context peaked at the appropriate $S+$ value. Presumably this withinsubject test procedure increased the saliency of the contextual cues during the test; their strong association with the two problems must have been formed during training, since generalization testing was carried out in extinction. The increased salience of contextual cues presumably led the subjects to follow the contextual instruction rather than the recency instruction.

Most research on long-term memory in animals has been primarily concerned with isolating the variables responsible for increasing or reducing PI and only indirectly with assessing the memory of the initial training. Among the variables that have been shown to favor recency (and thus reduce PI) are extensive training with distributed practice on the terminal problem (Burr \& Thomas, 1972; Spear, 1971), minimal training on the initial problem (Spear, 1971), and a long interval between training on the two problems (Spear, 1971). Spear (1971) has argued that these manipulations do not reduce the effect of prior learning on the retention of subsequent learning but, rather, postpone the manifestation of that effect. Thus, PI might be observable in a test delayed only $1 \mathrm{~h}$ following minimal active avoidance training, but would be apparent only after $24 \mathrm{~h}$ if testing followed extensive active avoidance training. The implication of this proposal is that the implicit recency instruction weakens with the passage of time.

Having shown that Context 1 (the context in which the initial discrimination was learned) could effectively retrieve the memory of Problem 1 when the within-subject test procedure increased the salience of the context, we endeavored to show that we could produce the same effect, in an indirect way, by weakening the (competing) recency instruction by allowing considerable time to pass between training and testing. To this end, in Experiment 1, original and reversal training was carried out in two different contexts and generalization testing was carried out, in Context 1, after delays of 1 day (as in Thomas et al., 1981), 7 days, or 30 days.

\section{EXPERIMENT 1}

\section{Method}

Subjects. The subjects were 12 experimentally naive pigeons obtained from a local supplier and maintained at $75 \%$ of their ad-lib weight. They were housed in individual cages in a colony room with a 16-h/8-h light/dark cycle.

Apparatus. The apparatus was the same as that employed by Thomas et al. (1981). Key stimuli were produced by IEE projectors (Model 00010-01-3043-1815) with No. 1815 lamps operated at $12.5 \mathrm{~V}$. Kodak Wratten filters, Nos. 75, 74, 99, 73, and 72B, produced nominal peak-transmitted wavelengths of $490,538,555$, 576 , and $606 \mathrm{~nm}$, respectively. The houselight was a No. 1829 bulb operated at $24 \mathrm{~V}$ behind a 3.8-cm-diam light-diffusing Plexiglas disk in the extreme upper right-hand corner of the intelligence panel. A standard electronic tone generator provided white noise or a 1,000-Hz tone at $80 \mathrm{~dB}$ SPL (re: .0002 dynes $/ \mathrm{cm}^{2}$ ). Exhaust fans provided masking noise. Reinforcement was $3 \mathrm{sec}$ of access to the food hopper, which was filled with mixed grain. All recording and control were accomplished with standard relay circuitry in an adjoining room.

Procedure. All subjects experienced the same training regimen. Throughout Phase 1, the context (Context 1) consisted of houselights-on plus the tone (HL T). On Day 1, all subjects were magazine trained, hand shaped to peck the 538-nm keylight, and allowed to earn 60 reinforcers on a gradually increasing variable interval (VI) schedule. On Day 2, the subjects were given singlestimulus training with the 538-nm keylight until 40 reinforcers were earned on a VI 30-sec schedule. Starting on Day 3, discrimination training was given, during which responding was reinforced on a VI 30-sec schedule during $S+$ trials and extinguished during S- trials. The subjects were run daily in 30-min sessions composed of three blocks of five S+ (538-nm) and five S- (576$\mathrm{nm})$ trials. Trials were of $57 \mathrm{sec}$ duration and separated by 3-sec blackouts, in a standard Gellerman series. The subjects were run on this task until $90 \%$ of their total responses were made in the presence of the $\mathrm{S}+$ during a single session. When each subject reached criterion, it was shifted to Phase 2 discrimination training on the following day. Phase 2 employed a reversal of the original problem; that is, $576 \mathrm{~nm}$ was $\mathrm{S}+$ (reinforced on a VI $30-\mathrm{sec}$ schedule), whereas $538 \mathrm{~nm}$ signaled extinction. In Phase 2, all subjects were run in a single session to a criterion of $90 \%$ of total 
responses to the $S+$ for a block of 10 trials. Trials and blackouts were of the same duration as in Phase 1. The context present (Context 2) was houselights-off plus the white noise ( $\overline{\mathrm{HL}} \mathbf{N})$.

The subjects were divided into three groups $(n=4)$, matched for discrimination performance, and were tested for wavelength generalization in extinction on the next day, 7 days later, or 30 days later. Testing took place in the same context in which the first discrimination problem was learned (HL T). The five test stimuli, 490, 538, 555, 576, and $606 \mathrm{~nm}$, were randomized into blocks, and 12 different randomized blocks were presented to each subject. Stimulus presentations were of $30 \mathrm{sec}$ duration and separated by 5 -sec blackouts.

\section{Results and Discussion}

Groups 1,2 , and 3 required a mean of $2.75,3.0$, and 3.0 sessions, respectively, to achieve criterion on the original problem. These means did not differ reliably $[F(2,9)<1]$. There were also no significant differences in the time required to master the reversal problem. Group 1 took a mean of $70.0 \mathrm{~min}$ compared with 75.0 and $77.5 \mathrm{~min}$ in Groups 2 and 3, respectively $[\mathbf{F}(2,9)<1]$.

For each subject a relative generalization gradient was computed based on the percent of total responses emitted to each of the five test stimuli. Figure 1 presents these individual gradients for the four subjects in each group. The results for Group 1 replicate those obtained in the original Thomas et al. (1981) experiment. As in that experiment, two of the gradients

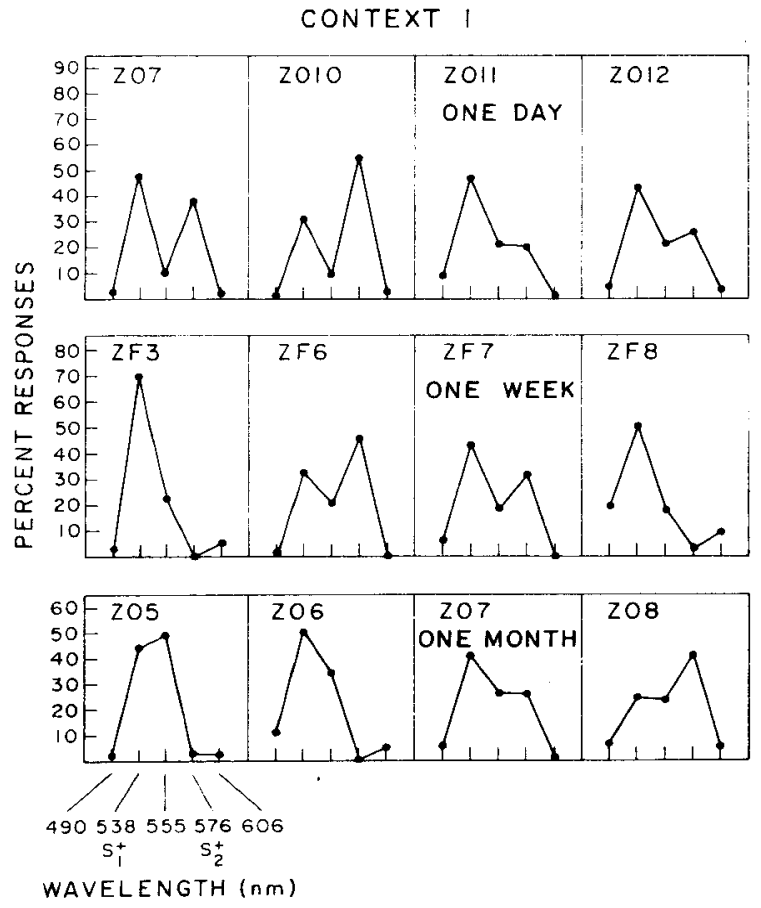

Figure 1. Relative wavelength generalization gradients of subjects tested in Context 1 after delays of 1 day (top row), 1 week (middle row), or 1 month (bottom row). S+, refers to the reinforced stimulus in original discrimination training; $S+t_{2}$ refers to the reinforeed stimulus during reversal training. clearly peaked at $538 \mathrm{~nm}$, indicating effective retrieval of the discrimination problem learned in Context 1 . Indeed, these two gradients, like those two obtained under a similar condition in the original experiment, were indistinguishable from those of control subjects (in the original Thomas et al., 1981, experiment), who had learned only the 538-nm S+, 576-nm Sproblem. On the other hand, the other two gradients, like the others in the original study, suggest a conflict between behavior controlled by recency and that controlled by the test context.

As the figure indicates, the same pattern of results was obtained in Groups 2 and 3. In each case, only two of the four gradients showed effective retrieval of the memory of Problem 1 during testing in Context 1. Thus, the prediction that the effectiveness of Context 1 as a retrieval cue would increase as testing was further delayed was not supported.

There are two plausible interpretations of this null effect. One is that, contrary to expectations, the recency effect, that is, the tendency of the subjects to perform as they last did in training, does not weaken with the passage of time, at least up to 30 days. Alternatively, recency may weaken but so may the memory of the association between Context 1 and Problem 1. If so, a weakened contextual instruction may compete no more effectively with a weakened recency instruction than did a strong context instruction against a strong recency instruction. Experiment 2 was designed to determine whether the association between each problem and its associated context weakened measurably during a 30-day period. To test for this possibility, a test procedure used by Thomas et al. (1981, Experiment 2) was employed. As in Experiment 1, the original discrimination and its reversal were learned in two different contexts, HL $\mathbf{T}$ and $\overline{\mathbf{H L}} \mathbf{N}$. In generalization testing, carried out after a 30-day delay, both contexts were presented in alternating fashion, permitting a gradient to be derived based on responding in each context. In addition to the original experiment by Thomas et al. (1981), this procedure was used by Thomas, McKelvie, and Mah (1983) and in several additional unpublished studies in our laboratory, all using a 1-day delay between training and testing. In almost every case, the gradients have shown excellent contextual control over the location of the gradient peaks by both Context 1 and Context 2 . In the present experiment, we asked whether the same would be true when 30 days intervened between training and testing.

\section{EXPERIMENT 2}

\section{Method}

Subjects. The subjects were four experimentally naive pigeons, maintained as in Experiment 1.

Apparatus. The apparatus was the same as that used in Experiment 1 . 
Procedure. The training procedure, the contexts, and discrimination problems were the same as those used in Experiment 1. All four subjects were tested for wavelength generalization in extinction 30 days after the completion of their training. As in Experiment 1 , the five test wavelengths were randomized into blocks, and 12 different random blocks were presented to each subject. The context was changed, however, after each pair of blocks. This was done in a counterbalanced fashion such that, for half of the subjects, Blocks 1 and 2, 5 and 6, and so on, were experienced in Context 1 (HL T), and, for the other subjects, they were experienced in Context 2 ( $\overline{\mathrm{HL}} \mathrm{N}$ ). Durations of stimulus presentations and blackouts were the same as in Experiment 1.

\section{Results and Discussion}

The performance of the subjects in discrimination training was comparable to that of subjects trained under the same conditions in Experiment 1.

Figure 2 presents the relative generalization gradients of each subject in this experiment. For three of the four subjects, each context was an effective retrieval cue and the two gradients peaked at the $\mathrm{S}+$ value associated with each. The fourth subject, YA 1 performed in an aberrant manner; its gradients both peaked at $538 \mathrm{~nm}$, the Problem $1 \mathrm{~S}+$, regardless of the context present. We have run more than 30 subjects in within-subject alternating context tests in our laboratory and virtually all have shown strong conditional control by each context, as three of the four subjects have done here. It seems reasonable to conclude that the lack of an effect in Experiment 1 is not attributable to a reduction in the potential of Context 1 to retrieve the memory of Problem 1 during the delay interval.

In Experiment 3, the hypothesis was tested that the recency effect, that is, the tendency of subjects to perform as they did last in training does not weaken over the 30-day period. This suggestion was tested directly by training the subjects on the original and reversal problems in the same context. Testing was carried out, as in Experiment 1, after 1, 7, and 30 days in different groups. Since the text context was present during the training of both problems, it should have no influence on the relative likelihood of retrieving the memories of the two conflicting problems. This permitted an assessment of the recency effect uncontaminated by context effects.

\section{EXPERIMENT 3}

\section{Method}

Subjects. The subjects were 12 experimentally naive pigeons, maintained as in Experiment 1.

Apparatus. The same apparatus was used as in Experiment 1.

Procedure. The training procedure and discrimination problems were the same as those used in Experiment 1, with the exception that the context present throughout training was HL T. Again, the subjects were divided into three groups for the purpose of wavelength generalization testing in extinction 1 day (Group 1), 7 days (Group 2), or 30 days (Group 3 ) after the completion of training. As in Experiment 1, the five test wavelengths were randomized into blocks, and 12 different random blocks were presented to each subject. The durations of stimulus presentations and blackouts were the same as in Experiment 1, but the context present throughout training continued to be present during generalization testing.

\section{Results and Discussion}

Groups 1, 2, and 3 required a mean of $3.25,3.25$, and 3.75 sessions, respectively, to achieve criterion on the original problem $[F(2,9)<1]$. There were also no significant differences in the time required to master the reversal problem. Group 1 took a mean of $97.5 \mathrm{~min}$, as compared with 87.5 and $102.5 \mathrm{~min}$ in Groups 2 and 3 , respectively $[F(2,9)<1]$. These

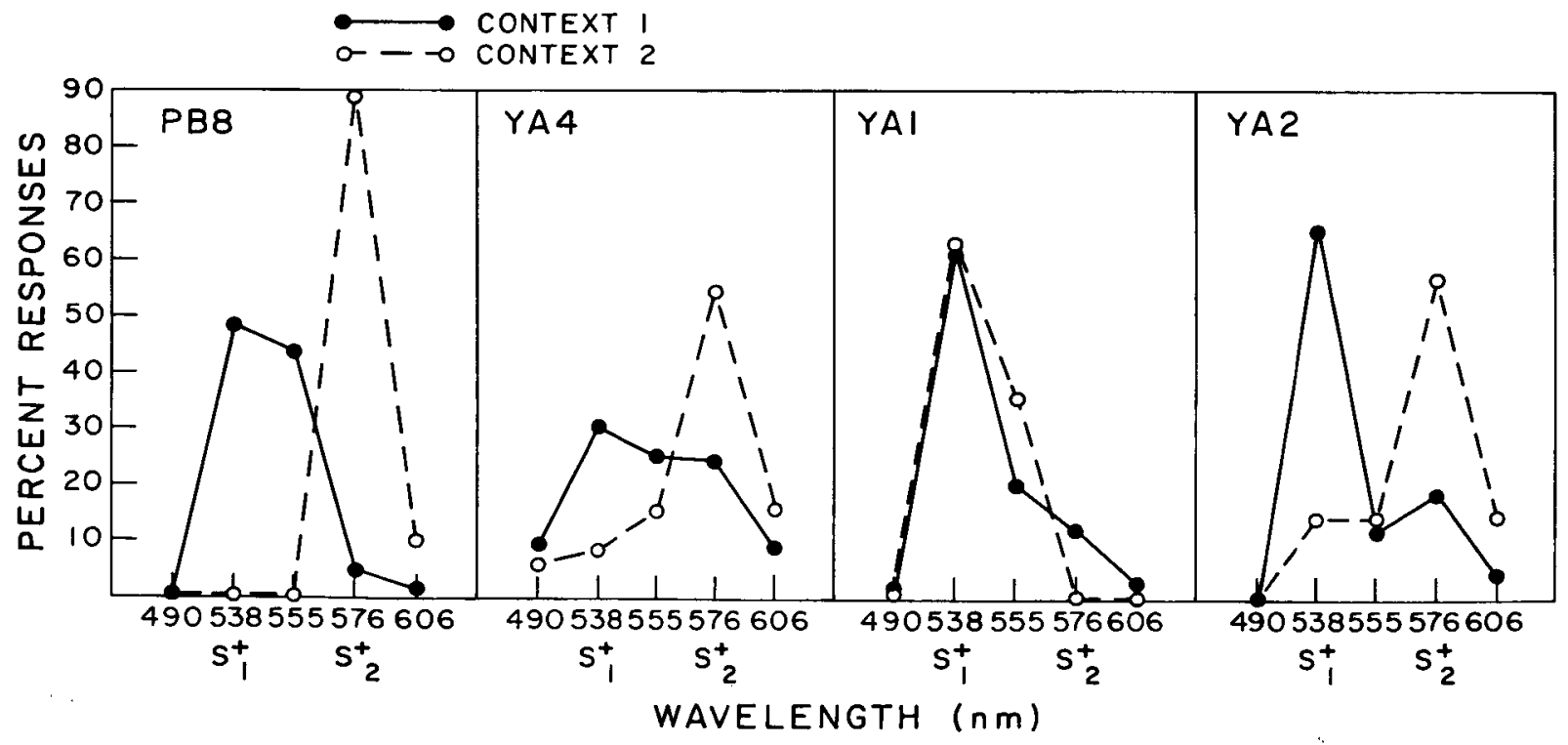

Figure 2. Relative wavelength generalization gradients of subjects trained on the original and the reversal problems in different contexts and tested in both contexts after a delay of 1 month. 
times were longer, however, than required by the groups in Experiments 1 and 2, who learned the original and reversal problems in different contexts. This same difference was reported by Thomas et al. (1981) as well as by Thomas et al. (1983). The explanation would appear to be that when the reversal is learned in the same context as the original problem, the context strengthens the memory of Problem 1 during the learning of Problem 2, thereby increasing negative transfer.

Figure 3 presents the relative generalization gradients of the four subjects in each group. As in the Thomas et al. (1981) experiment, in Group 1 (24-h delay), all gradients showed recency, peaking at $576 \mathrm{~nm}$, the S+ from Problem 2. The same was true for Group 2, even though this group was tested 7 days after training, and even in Group 3, tested after 30 days, all gradients peaked primarily at $576 \mathrm{~nm}$, even though there was evidence of some flattening. These results, then, are consistent with those of Experiment 1 . The recency effect is still evident after 7 and $\mathbf{3 0}$ days; hence, testing in a context unique to Problem 1 in Experiment 1 after these time periods was no more effective than was testing after 1 day.

In comparison with the rat literature (cf. Spear, $1971,1978)$ the experiments described here have indicated surprisingly little effect of temporal parameters on the strengths of contextual and recency "instructions." The differences may not be due to species,

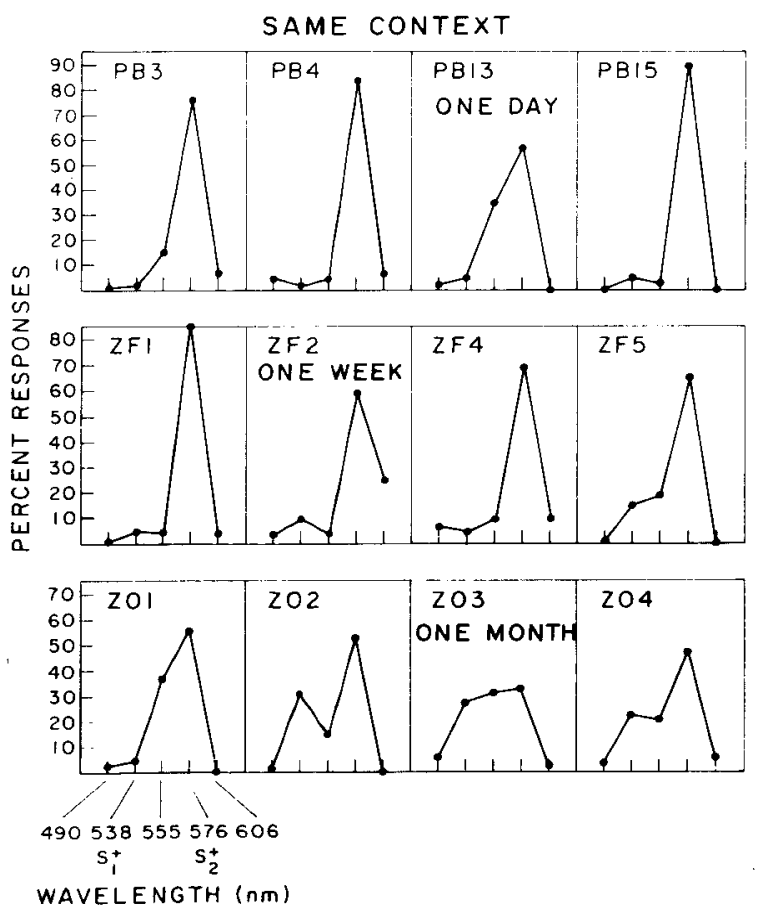

Figure 3. Relative wavelength generalization gradients of subjects trained on both problems in the same context and tested in that context afier delays of 1 day (top row), 1 week (middle row), or 1 month (bottom row). however, since the procedures and the measures used are so different. All of our experiments have used discrimination training tasks. In the rat memory literature, interference effects have more frequently been observed in simple go/no-go tasks, and Gleitman (1971) has reported the failure to find simple forgetting with the passage of time following discrimination training unless an explicit source of PI (i.e., former training on a conflicting problem) was provided. Both Gleitman (1971) and Spear (1971) have speculated that PI may reflect forgetting of the sequential order of the two learned tasks. Perhaps our pigeons show so little PI because the original and reversal discriminations are easily kept distinct because they are learned in different ways or are qualitatively different tasks. One procedural difference that can be shown to be irrelevant is the fact that, in these experiments, the original discrimination was learned over several sessions (i.e., with distributed practice), whereas the reversal was learned in a single session (i.e., with massed practice). In several unpublished studies, we have obtained equally strong evidence for the recency effect when both problems were learned with distributed practice. Another potentially significant factor is the fact that the most recent learning involves a reversal, hence the extinction of a previously reinforced response tendency, whereas acquisition of Problem 1 does not. In Experiment 4, we asked whether a recency effect would be obtained and whether it would persist over time if both the final problem and the penultimate problem involved reversal learning. This was accomplished by giving subjects training on three reversals rather than just one. As in the previously described experiments, the terminal problem was 576-nm S+, 538-nm S-, but the immediately preceding problem had also been learned as a reversal of the problem that had immediately preceded it.

\section{EXPERIMENT 4}

\section{Method}

Sabjects. The subjects were 12 experimentally naive pigeons, maintained as in Experiment 1.

Apparatus. The apparatus was the same as that used in Experiment 1.

Procedure. All subjects experienced the same training regimen. Pretraining and Phase 1 were the same as in the other experiments, and, as in Experiment 3, the same context (HL T) was used throughout. When the subjects achieved a discrimination criterion of $90 \%$ of total responses to the $\mathbf{S}+$ on the initial problem during a session (538-nm S+, 576-nm S-), they were switched to the reversal on the next day. Unlike in the other experiments, however, Phase 2 employed daily $30-\mathrm{min}$ sessions and was continued until the same criterion was reached. Phase 3 involved a second reversal and Phase 4 involved a third, so the terminal problem (i.e., that in Phase 4) was 576-nm S+, 538-nm S-.

The subjects were divided into three groups $(n=4)$ on the basis of overall discrimination performance and they were tested for wavelength generalization in extinction 1,7 , or 30 days after the completion of Phase 4 training. The training context (HL T) was 
present during testing, which employed the same format as in the other experiments.

\section{Results and Discussion}

The mean number of sessions required to achieve criterion on Problem 1 was 3.2 sessions, as compared with 5.25, 6.75, and 7.0 sessions on Problems 2, 3, and 4 , respectively. This effect of problem was significant $[F(3,44)=4.06, p<.05]$, and the difference is largely attributable to the fact that the initial problem was mastered more quickly than were any of the reversals. In addition, there is no evidence to suggest any improvement in reversal performance with accumulating experience. This agrees with a study by Miller, Hansen, and Thomas (1972), which showed improvement when training on each problem was terminated when a rather lax criterion (i.e., $80 \%$ of total responses to $\mathrm{S}+$ ) was achieved but not when a rigorous criterion (i.e., $95 \%$ of responses to $\mathrm{S}+$ ) was employed. Studies of serial reversal learning which show substantial improvement after many reversals typically employ a discrete trial procedure and reverse problems after every one or two sessions when performance is still at a relatively low level (cf. Bitterman, 1969; Mackintosh, 1969).

Figure 4 presents the relative generalization gradients of the four subjects in each group. As may be seen, the gradients of all subjects show recency, peak-

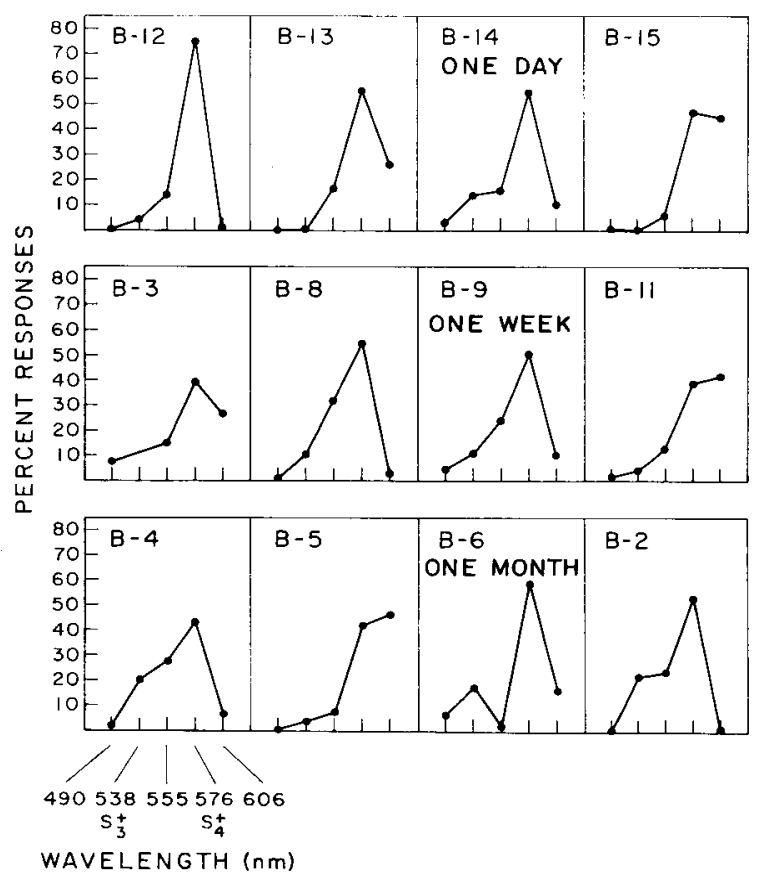

Figure 4. Relative wavelength generalization gradients of subjects that received serial reversal training in the same context and were tested in that context after delays of 1 day (top row), 1 week (middle row), or 1 month (bottom row). $\mathrm{S}+4$ refers to the reinforced stimulus during the terminal problem; $\mathrm{S}+3$ was the rein. forced stimulus during the problem that immediately preceded it. ing at $567 \mathrm{~nm}$, the $\mathrm{S}+$ of the terminal problem, or at $606 \mathrm{~nm}$, a value still farther removed from the final S-. Apparently, the repeated reversal training enhances the displacement of the gradient peak following intradimensional discrimination training. Peak shifts were not observed in the earlier experiments and were not anticipated in this one in view of the wide spacing of both the training and the generalization test values. A gradient peak at 576 or $606 \mathrm{~nm}$ however, reflects the recency effect, which is still clear in subjects tested after a 30-day delay. This result is surprising in view of the fact that both the terminal and the penultimate problems were reversals of prior problems and thus should have been qualitatively similar.

The use of several reversals, designed to enhance forgetting of the sequential order of the two conflicting habits, did not result in an enhanced tendency to respond to the original $\mathrm{S}+$. On the other hand, this manipulation had a clear effect on the slope of the obtained gradients; they tended to be flatter than those obtained in Experiment 3. In an experiment reported by Thomas et al. (1981, Experiment 1), despite a recency effect reflected in gradients that peaked at the $\mathrm{S}+$ of the terminal problem, PI was revealed in gradients that were flatter in subjects that had experienced a reversal than in subjects that had not. Thomas (1981) has proposed that the location of the gradient peak may help to identify the target memory, that is, it may indicate what is being remembered, whereas the slope of the gradient may indicate how well the target memory has been retrieved. If we reconsider the results of both Experiment 3 and Experiment 4 in this light, two interesting findings are revealed. With the generalization slope measure, there is more forgetting (and thus a weaker recency effect) after three reversals than after one, and there is a progressive weakening of recency with the passage of time.

For purposes of statistical analysis, the percent of total responses that each subject gave to $576 \mathrm{~nm}$ served as an index of gradient slope. A $3 \times 2$ analysis of variance was performed to examine the effects of the different delay intervals (1 day vs. 1 week vs. 1 month) and those of one reversal versus three. Both main effects were highly significant. The mean percentages to $576 \mathrm{~nm}$ were 68,58 , and 48 after 1,7 , and 30 days, respectively $[F(2,18)=6.05, p<.01]$. The mean percentage to $576 \mathrm{~nm}$ after one reversal was 64 , as compared with $51 \%$ after three reversals $[F(1,18)=8.49$, $\mathrm{p}<.01]$. The interaction was not significant $[\mathrm{F}(2,18)$ $=3.13]$. The effect of the different delay intervals is particularly interesting in view of the fact that we have previously found no changes in wavelength gradient slope subsequent to 1 day's delay following single-stimulus training (Thomas \& Lopez, 1962) or following experience with a single-discrimination problem (Thomas, Ost, \& Thomas, 1960). However, 
when one or several reversals provide a source of PI, as in the present study, forgetting continues to increase over a period of at least a month.

The use of the generalization slope measure reconciles what appeared to be a discrepancy between these results and those reported by Spear (1971) with rats. In Spear's study, PI increased with increasing trainingtest delays. In Spear's paradigm, responding that was inappropriate to the terminal problem was attributed to recovery of the strength of the memory of the initial problem. This interpretation may be gratuitous, however, because, when only two responses are possible, how can one distinguish between a decrease in the strength of Response 2 and an increase in the strength of Response 1? In the paradigm employed here, the observed interference is not simply a consequence of the strengthening of responding to a particular alternative stimulus. Rather, it seems to reflect confusion or uncertainty as to what the terminal training stimulus was. The finding of greater forgetting with three reversals than with one is certainly consistent with this view. On the other hand, the fact that gradients continue to peak at the terminal $\mathrm{S}+$, even after three reversals and after long delays, suggests that the "intent" to perform as subjects did last is still present, even though the memory of the terminal S + value has measurably weakened.

\section{GENERAL DISCUSSION}

In Experiments 1 and 2, testing subjects in a context that had been uniquely associated with original training either partially (Experiment 1) or completely (Experiment 2) overcame the recency effect. It accomplished this by facilitating the retrieval of an alternative habit, that of responding to green, the original S + . This finding is consistent with the view taken by Spear $(1971,1978)$ and Thomas et al. (1981) that interference in long-term memory in animals is fruitfully viewed as a retrieval problem. On the other hand, the interference observed in Experiments 3 and 4 cannot be as easily characterized. There is no evidence of an enhanced tendency to respond in accordance with original training but only of a reduced tendency to respond in accordance with terminal training. The interference is nonspecific and therefore of a different sort from that affected by context manipulations. Perhaps it can be characterized as increasing uncertainty as to what the value of the terminal S+ was. These results are consistent with those of a recent study from Spear's laboratory (cf. Spear, Smith, Bryan, Gordon, Timmons, \& Chiszar, 1980), which showed increasing PI with increasing delays, but did not find any systematic increase in the effectiveness of contextual retrieval cues (cf. Experiment 2).

The question remains of how best to explain the recency effect per se. Consistent with his position on interference, Spear (1971) has proposed that recency can be viewed as a retrieval phenomenon; that is, the changing internal and external stimuli that serve as retrieval cues at the time of the test would ordinarily be more similar to those present during the most recent learning than to those present at an earlier point in time. Although such differences might account for recency effects over a short time period, it is difficult to see how they could be functional after a 30-day delay.

An alternative retrieval explanation asserts that the association between stable contextual cues (e.g., the sights, sounds, and smells of the experimental chamber) and the initial problem is extinguished during the learning of the terminal problem. Thus, those contextual cues are associated only (or at least primarily) with the terminal problem by the time training is completed. When those cues are present during testing, it is the memory of the terminal problem that is therefore activated.

Still another way to view recency is to suggest that it represents a strategy that subjects adopt based on their past experience in the real world and/or the experimental laboratory. Since contingencies tend to remain in effect for reasonably extended periods of time, it may prove profitable for subjects to assume that the contingencies that were last in effect remain in effect.

The more interesting experimental question may be to ask why the recency effect should ever dissipate with time. The traditional explanation was in terms of the spontaneous recovery of the previously extinguished habit; however, Gleitman (1971) has pointed out that the terminal course of spontaneous recovery is inappropriate to account for observed PI. Maier, Allaway, and Gleitman (1967) also determined, in a direct test, that spontaneous recovery plays no role in the amount of PI obtained following a discrimination reversal in rats. In their study, PI was independent of whether or not latest learning involved extinction of an originally correct response. In the present study (Experiments 3 and 4), although PI increased with the passage of time up to 1 month, it could not be attributed to a recovery in the strength of the initial discrimination habit. Rather, the interference effect was nonspecific in nature. Clearly the inclusion of a source of PI in these studies enhances forgetting as revealed in flattened generalization gradients, but the mechanism by which it does so remains to be determined.

\section{REFERENCES}

Bitterman, M. E. (1969). Habit reversal in probability learning: Rats, birds, fish. In R. M. Gilbert \& N. S. Sutherland (Eds.), Animal discrimination learning. New York: Academic Press.

Burn, D. E. S., \& Thomas, D. R. (1972). Effect of proactive inhibition upon the post-discrimination generalization gradient. Journal of Comparative and Physiological Psychology, 81, 441- 448 . 
Gleitman, H. (1971). Forgetting of long-term memories in animals. In W. K. Honig \& P. H. R. James (Eds.), Animal memory. New York: Academic Press.

Mackintosh, N. J. (1969). Comparative studies of reversal and probability learning: Rats, birds, fish. In R. M. Gilbert \& N. S. Sutherland (Eds.), Animal discrimination learning. New York: Academic Press.

Maier, S. F., Allaway, T. A., \& Gleitman, H. (1967). Proactive inhibition in rats after prior partial reversal: $A$ critique of the spontaneous recovery hypothesis. Psychonomic Science, 9, 63-64.

Miller, J. T., Hansen, G., \& Thomas, D. R. (1972). Effects of stimulus similarity and response criterion on successive discrimination reversal learning. Journal of Comparative and Physiological Psychology, 81, 434-440.

SPEAR, N. E. (1971). Forgetting as retrieval failure. In W. K. Honig \& P. H. R. James (Eds.), Animal memory. New York: Academic Press.

SPEAR, N. E. (1978). The processing of memories: Forgetting and retention. Hillsdale, $\mathrm{NJ}$ : Erlbaum.

Spear, N. E., Smith, G. J., Bryan, R. G., Gordon, W. C., Timmons, R., \& Chiszar, D. A. (1980). Contextual influences on the interaction between conflicting memories in the rat. Animal Learning \& Behavior, 8, 273-281.

Thомаs, D. R. (1981). Studies of long-term memory in the pigeon. In N. E. Spear \& R. R. Miller (Eds.), Information processing in animals: Memory mechanisms. Hillsdale, NJ: Erlbaum.

Thomas, D. R., McKelvie, A. R., \& MaH, W. L. (1983). The context as a conditional cue in operant discrimination reversal learning. Manuscript submitted for publication.

Thomas, D. R., \& Lopez, L. J. (1962). The effect of delayed testing on generalization slope. Journal of Comparative and Physiological Psychology, 44, 541-544.

Thomas, D. R., McKelvie, A. R., Ranney, M., \& Moye, T. B. (1981). Interference in pigeons' long-term memory viewed as a retrieval problem. Animal Learning \& Behavior, 9, 581-586.

Thomas, D. R., Ost, J., \& Thomas, D. H. (1960). Stimulus generalization as a function of the time between training and testing procedures. Journal of the Experimental Analysis of Behavior, 3, 9-14.

(Manuscript received June 10, 1983; accepted for publication October $17,1983$. ) 